\title{
Desigualdades sociais e os desafios à estratégia de eliminação da tuberculose no Brasil
}

\author{
Regina Célia Fiorati ${ }^{\mathrm{a}^{*}}$, Fernanda Carla de Assis Cândido ${ }^{\mathrm{b}}$, Larissa Barros de Souza ${ }^{\mathrm{b}}$, \\ Marcela Paschoal Popolin ${ }^{\mathrm{b}}$, Antônio Carlos Vieira Ramos ${ }^{\mathrm{b}}$, Ricardo Alexandre \\ Arcêncio ${ }^{\mathrm{b}}$
}

${ }^{a}$ Faculdade de Medicina de Ribeirão Preto, Universidade de São Paulo-USP, Ribeirão Preto, SP, Brasil

${ }^{\mathrm{b}}$ Escola de Enfermagem de Ribeirão Preto, Universidade de São Paulo-USP, Ribeirão Preto, SP, Brasil

Histórico do Artigo

Recebido em

21/11/2017

Aceito em

$27 / 03 / 2018$

Palavras-chave:

Tuberculose;

Determinantes Sociais;

Vulnerabilidade Social;

Desigualdade Social; Iniquidade Social.

\begin{abstract}
RESUMO
O presente trabalho teve como objetivo identificar o impacto das desigualdades sociais na cadeia de progressão da tuberculose no Brasil visando à identificação dos nós críticos à eliminação da doença. Foi realizada uma revisão de literatura dos artigos disponíveis nas bases de dados Cummulative Index to Nursing and Allied Health Literature, Literatura Internacional em Ciências da Saúde e Literatura Latino-Americana e do Caribe em Ciências da Saúde, nos idiomas português, inglês e espanhol. Como critério de inclusão, selecionaram-se artigos publicados na íntegra cujo cenário de investigação fosse o Brasil, no período de 2005 a 2015. Utilizaram-se os descritores do Medical Subject Headings, de acordo com as combinações: Access or accessibility and Tuberculosis; social determinants of health and tuberculosis; social vulnerability and tuberculosis; social inequity or social inequality and tuberculosis. A análise dos estudos selecionados pautou-se no referencial de Melnyk, Fineout-Overholt por meio da análise da qualidade do nível de evidência científica dos artigos selecionados com base na Classificação dos Níveis de Evidências Científicas. Foram analisados 45 artigos, os quais mostraram os principais determinantes sociais da saúde que representam barreiras à meta End $T B$, sendo eles: as condições econômicas, seguidas pela acessibilidade a serviços, violação de direitos e estilos de vida, fatores que impactam na cadeia de progressão da tuberculose. Conclui-se que desigualdades sociais são barreiras consideráveis à eliminação da doença, portanto intervenções de amplo espectro devem ser consideradas. Os desafios à sociedade brasileira ainda são substanciais e devem envolver para além do setor da saúde, os setores ligados à gestão pública nas várias esferas governamentais.
\end{abstract}

Social inequality and the challenges to TB elimination strategy in Brazil
Keywords:

Tuberculosis; Social

Determinants; Social Vulnerability; Social inequality; Social Inequity.

\begin{abstract}
The objective of the present wok was to identify the impact of social inequalities in the chain of tuberculosis progression in Brazil, aiming to identify the nodes critical to the elimination of the disease. It was carried out literature review of articles available in Cummulative Index to Nursing and Allied Health Literature, International Literature in Health Sciences and Latin American and Caribbean Literature in Health Sciences, Portuguese, English and Spanish. As inclusion criterion, we selected articles published in full whose research scenario is Brazil, from 2005 to 2015. The descriptors of the Medical Subject Headings were used according to the combinations: Access or accessibility and Tuberculosis; social determinants of health and tuberculosis; social vulnerability and tuberculosis; social inequality or social inequality and tuberculosis. The analysis of the selected studies was based on the Melnyk, Fineout-Overholt reference by means of the analysis of the quality of the level of scientific evidence of the articles selected based on the Classification of Levels of Scientific Evidence. Fourthy five articles were analyzed, which showed the main social determinants of health that represent barriers to the End TB goal, being: economic conditions, followed by accessibility to services, violation of rights and lifestyles, factors that impact on the chain of progression of tuberculosis. In summary, social inequalities are considerable barriers to the elimination of the disease, so broad-spectrum interventions must be considered. Challenges to Brazilian society are still substantial and should involve, beyond the health sector, the sectors related to public management in the various governmental spheres.
\end{abstract}

\footnotetext{
* Autor para correspondência: reginacf@fmrp.usp.br (Fiorati R. C.)
} 


\section{Introdução}

A tuberculose (TB) constitui um problema de saúde pública em escala global, afetando principalmente 30 países (High Burden Countries - HBC) que concentram $84 \%$ da carga da doença no mundo. O Brasil ocupa a $20^{\mathrm{a}}$ posição, apresentando incidência de 41 casos por 100.000 habitantes e mortalidade de 2,7 óbitos para cada 100.000 habitantes (1).

A Organização Mundial da Saúde (OMS), em 2015, aprovou a estratégia "End TB", que tem como objetivo a eliminação da TB ( $<1$ caso por 100.000 habitantes) e ainda a redução em 95\% das mortes por TB até 2035 (2). O Brasil é signatário da proposta (2), todavia um desafio que se coloca é a própria acessibilidade da população aos serviços de saúde consonante às suas necessidades em tempo, lugar, de forma justa e resolutiva (3).

Desde a publicação da estratégia, estudos têm sido conduzidos, evidenciando limites dos TB-HBC de alcançar as metas. De acordo com Huynh (4), dificilmente países como a China irá alcançá-la até 2035 se não incorporar em seu arsenal medidas de proteção social, como diagnóstico mais rápido, implementação de novas drogas e regimes terapêuticos, e intervenções focadas sobre fatores de risco para a TB como o tabagismo, diabetes e poluição do ar, e ações de saúde sobre populações em áreas densamente povoadas que recebem populações migrantes de outras regiões do país.

O estudo de Lönnroth e Raviglione (5) traz a importância da distribuição equitativa dos recursos de saúde e reforço à proteção social pelos sistemas locais de saúde e da justiça social enquanto política de Estado. O trabalho de Gesesew et al (6) evidencia a força dos determinantes sociais nas taxas de mortalidade por TB e HIV na Etiópia, ainda a interação delas com a falta de recursos ou insumos para diagnóstico e sua não alocação equitativa.

Uma investigação conduzida no Peru traz a força dos determinantes sociais no desenvolvimento da multidrogaresistência da TB (TB-MDR) (7), quando o paciente apresenta resistência à isoniaziada e rifampicina, com ou sem resistência às drogas de primeira linha (2).

Apesar da relevância do tema e do número de trabalhos produzidos sobre TB, desconhece-se no Brasil as limitações em alcançar as metas, considerando os determinantes sociais na cadeia de progressão da TB. Os determinantes estruturais são aqueles ligados a estratificação social e incluem os fatores socioeconômicos, trabalho e renda, gênero, cultura e capital social. Os intermediários incluem condições de vida, condições de trabalho, disponibilidade de alimentos, seguridade social, comportamentos da população e barreiras para a adoção de um estilo de vida saudável (8).

O contexto político é compreendido também como fatores determinantes, pois influenciam processos de saúde/doença da população. Por meio de políticas de Estado devem-se prover melhores condições de vida cotidianas, das circunstâncias em que as pessoas nascem, crescem, vivem, trabalham e envelhecem. Sob esse prisma, todas as pessoas precisam de proteção social ao longo do tempo, em particular, casos que sofram eventos específicos, como a $\mathrm{TB}$, que leva a perda de rendimento ou trabalho e mais pobreza (9-11).

Portanto, adotar a abordagem dos determinantes sociais significa compreender o valor que eles têm para a TB e admitir que a eliminação da doença depende de ações que perpassam o setor saúde. Uma vez que está posto o desafio pela OMS, é importante evidenciar os limites e ou potencialidades dos sistemas de serviços de saúde do Brasil em alcançar a Estratégia End TB.

O objetivo do estudo é identificar o impacto das desigualdades sociais na cadeia de progressão da tuberculose no Brasil visando à identificação dos nós críticos à eliminação da doença. 


\section{Materiais e métodos}

Trata-se de um estudo de revisão de literatura cuja questão norteadora da pesquisa foi: como estão configuradas as desigualdades sociais na cadeia de progressão da TB e os desafios à estratégia de eliminação da doença no Brasil?

De acordo com os procedimentos definidos por Sampaio, Mancini (12) e Malik (13) de sistematização da revisão de literatura, seguiu-se um rigoroso processo de busca e seleção de estudos por meio de um checklist de qualidade que comprovasse a validade científica e relevância dos estudos, considerando o objeto investigado.

É importante destacar que a revisão de literatura foi de orientação qualitativa, pois o estudo importou-se com uma questão cuja discussão de sua evidência científica se dá com base em processos de interpretação de temas conceituais, que vão se construindo com base em análise crítica e revisões de teses teóricas (12,13). Considerando tais aspectos, os autores recorreram ao instrumento SPIDER para avaliação do estudo em termos da amostra (Sample - S), fenômeno de interesse (Phenomenon - PI), desenho (Design - D), avaliação (Evaluation - E) e tipo de pesquisa (ResearchType - R) (14).

O estudo foi desenvolvido segundo as etapas de formulação do problema e da questão norteadora, levantamento de dados preliminares para definição dos descritores, busca em bases de dados, compilação dos dados coletados, avaliação do material para seleção da amostra final, leitura e avaliação da amostra final, apresentação e discussão dos resultados (15).

Para levantamento dos artigos na literatura, foram realizadas buscas no período de agosto a outubro de 2015 em três bases de dados: CINAHL (Cumulative Index to Nursing and Allied Health Literature), PubMed (US National Library of Medicine National Institute of Heath) e LILACS (Literatura Latino-Americana em Ciências da Saúde). O período de dez anos (2005 a 2015) corresponde à data de criação da Comissão dos Determinantes Sociais da Saúde (CSDH) pela Organização Mundial da Saúde (OMS), em 2005.

Os critérios de inclusão para a busca dos artigos foram: artigos que decorressem de estudos realizados no Brasil; publicados em português, inglês ou espanhol; estudos que trouxessem pelo menos um descritor em seu título/title/titulo ou resumo/abstract/resúmen, artigos de pesquisas com fontes primárias de dados e artigos cujos resultados mostraram os fatores relacionados ao impacto das desigualdades sociais na cadeia de progressão da TB.

Foram incluídos descritores válidos no MeSH (Medical Subject Headings) utilizados de acordo com as combinações: Access or accessibility and Tuberculosis; social determinants of health and tuberculosis; social vulnerability and tuberculosis; social inequity or social inequality and tuberculosis.

A análise dos estudos selecionados pautou-se em Melnyk, Fineout-Overholt (16) por meio da análise da qualidade do nível de evidência científica dos artigos selecionados com base na Classificação dos Níveis de Evidências Científicas (Quadro 1), sendo que a análise e a posterior síntese foram realizadas de forma descritiva, possibilitando a observação e a classificação temática de determinados eixos de coerência e nível de evidência dos estudos, reunindo, assim, o conhecimento produzido em relação à questão focal da pesquisa. Ademais, cabe ressaltar que a busca e análise dos dados dos estudos foi realizada pelos pesquisadores de forma independente. A análise do material empírico pautou-se no referencial de determinantes sociais definido pela OMS (10). 
Quadro 1: Classificação dos níveis de evidência científica

\begin{tabular}{|c|l|}
\hline Níveis & \multicolumn{1}{|c|}{ Força de Evidência } \\
\hline I & $\begin{array}{l}\text { Evidências são provenientes de revisão sistemática ou metanálise de todos relevantes } \\
\text { ensaios clínicos randomizados controlados ou oriundas de diretrizes clínicas baseadas em } \\
\text { revisões sistemáticas de ensaios clínicos randomizados controlados }\end{array}$ \\
\hline II & $\begin{array}{l}\text { Evidências derivadas de pelo menos um ensaio clínico randomizado controlado bem } \\
\text { delineado }\end{array}$ \\
\hline III & Evidências obtidas de ensaios clínicos bem delineados sem randomização \\
\hline IV & Evidências provenientes de estudos de coorte e de caso-controle bem delineados \\
\hline V & Evidências originárias de revisão sistemática de estudos descritivos e qualitativos \\
\hline VI & Evidências derivadas de um único estudo descritivo ou qualitativo \\
\hline VII & Evidências oriundas de opinião de autoridades e/ou relatório de comitês de especialistas \\
\hline
\end{tabular}

\section{Resultados}

$\mathrm{Na}$ primeira fase da busca dos artigos, foram utilizados os descritores e suas combinações. No total, foram encontrados 1674 artigos; sendo 875 artigos na base de dados da PubMed; 468 artigos na base de dados CINAHL e 331 artigos na base de dados LILACS. Para a fase de seleção e análise dos artigos (Quadro 2), prosseguiu-se a avaliação 179 dos títulos e resumo e leitura integral, conformando uma amostra de 45 artigos. A figura 1 apresenta o fluxograma da revisão de literatura.

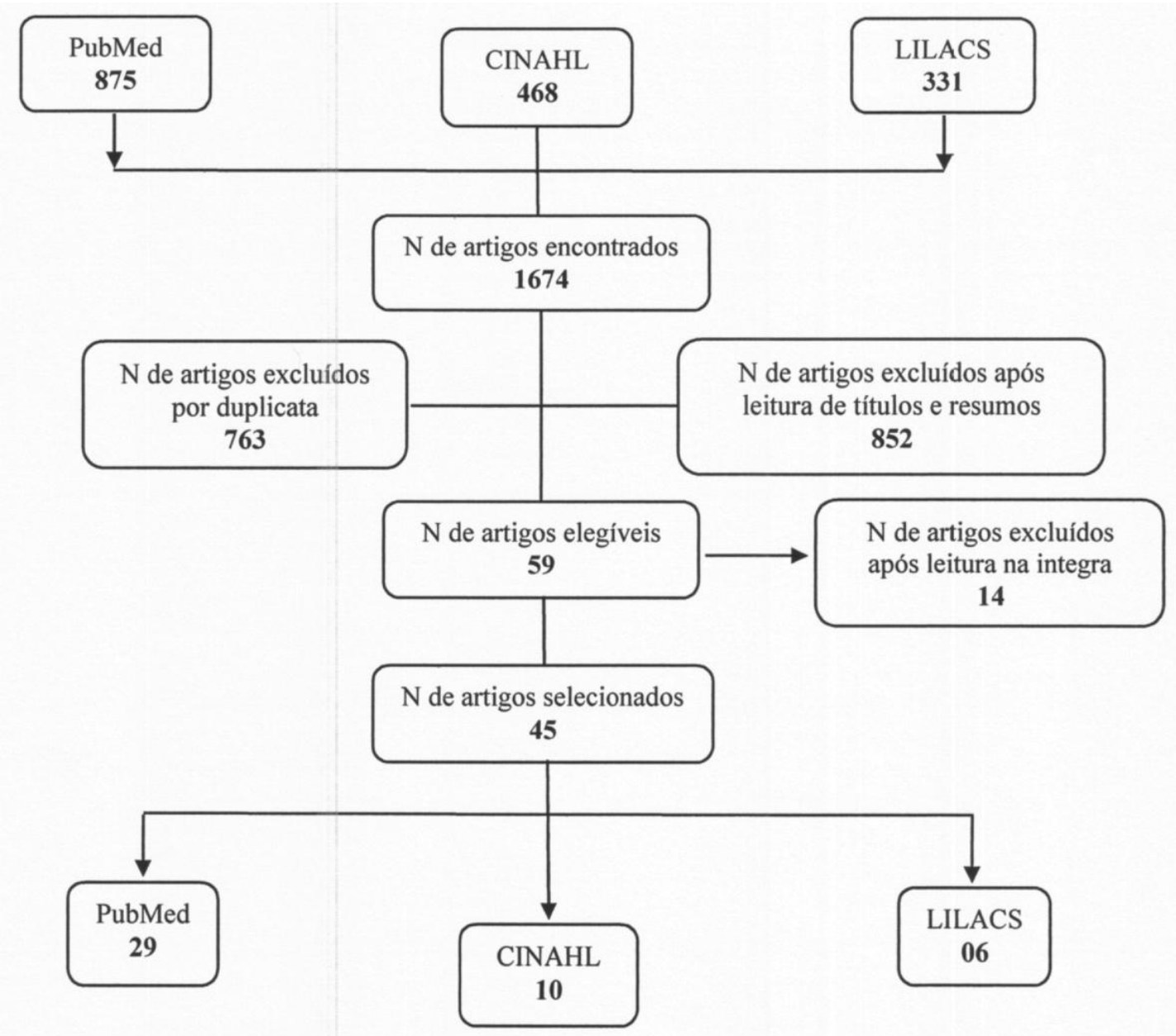

Figura 1: Fluxograma das etapas de revisão de literatura 
No processo de revisão dos trabalhos científicos, obteve-se a distribuição dos artigos considerando as bases de dados consultadas, que foram 10 a partir do CINAHL, seis da base LILACS e 29 da Pubmed (Quadro 2).

Quadro 2: Descritores utilizados na revisão de literatura

\begin{tabular}{|c|c|c|}
\hline Descritor & Base de dados & N \\
\hline \multirow{3}{*}{ Access or accessibility and TB } & Cinahl & 07 \\
\cline { 2 - 3 } & Pubmed & 12 \\
\cline { 2 - 3 } Social vulnerability or social risk and TB & Lilacs & 06 \\
\cline { 2 - 3 } & Cinahl & 01 \\
\cline { 2 - 3 } Social determinants and TB & Pubmed & 03 \\
\hline \multirow{3}{*}{ Social inequity or social inequality and TB } & Lilacs & 00 \\
\cline { 2 - 3 } & Cinahl & 01 \\
\cline { 2 - 3 } & Pubmed & 00 \\
\cline { 2 - 3 } & Lilacs & 00 \\
\hline \multirow{2}{*}{ Total } & Cinahl & 01 \\
\hline
\end{tabular}

Após a conformação da amostra final, foi confeccionado um quadro sinóptico (Quadro 3) com dados extraídos dos artigos, segundo o protocolo SPIDER.

Quadro 3: Quadro Sinóptico de extração dos estudos

\begin{tabular}{|c|c|c|c|c|c|c|c|}
\hline Ano & Macrorregião & Referência & Amostra (S) & $\begin{array}{l}\text { Fenômeno de } \\
\text { interesse (PI) }\end{array}$ & $\begin{array}{c}\text { Tipo de Estudo } \\
\text { (D) }\end{array}$ & $\begin{array}{l}\text { Avaliação } \\
\text { (nível de } \\
\text { evidência) } \\
\text { (E) }\end{array}$ & $\begin{array}{l}\text { Orientação } \\
\text { (R) }\end{array}$ \\
\hline 2005 & Brasil & $\begin{array}{l}\text { Garnelo L; } \\
\text { Brandão LC; } \\
\text { Levino A }\end{array}$ & Indígenas & $\begin{array}{c}\text { Acessibilidade } \\
\text { dos serviços }\end{array}$ & $\begin{array}{l}\text { Estudo } \\
\text { ecológico }\end{array}$ & IV & Quantitativo \\
\hline 2005 & Nordeste & Santos et al. & $\begin{array}{l}\text { Pacientes } \\
\text { com TB }\end{array}$ & $\begin{array}{c}\text { DSS - } \\
\text { econômicos }\end{array}$ & Estudo de coorte & IV & Quantitativo \\
\hline 2006 & Nordeste & $\begin{array}{l}\text { Cavalcanti } \\
\text { et al. }\end{array}$ & Idosos & $\begin{array}{l}\text { DSS - } \\
\text { estilo de vida }\end{array}$ & $\begin{array}{c}\text { Estudo de } \\
\text { coorte e de } \\
\text { caso-controle }\end{array}$ & IV & Quantitativo \\
\hline 2007 & Sudeste & Santos et al. & $\begin{array}{l}\text { Populações } \\
\text { vulneráveis }\end{array}$ & $\begin{array}{c}\text { DSS - } \\
\text { econômicos }\end{array}$ & Epidemiológico & IV & Quantitativo \\
\hline 2007 & Sudeste & Souza et al. & $\begin{array}{c}\text { Profissionais } \\
\text { da saúde }\end{array}$ & $\begin{array}{c}\text { DSS - } \\
\text { econômicos }\end{array}$ & - & VI & Qualitativo \\
\hline 2007 & Norte & $\begin{array}{l}\text { Levino; } \\
\text { Oliveira }\end{array}$ & $\begin{array}{c}\text { População } \\
\text { indígena }\end{array}$ & $\begin{array}{c}\text { Acessibilidade } \\
\text { aos serviços }\end{array}$ & $\begin{array}{c}\text { Estudo seccional } \\
\text { transversal }\end{array}$ & IV & Quantitativo \\
\hline 2007 & Nordeste & $\begin{array}{c}\text { Albuquerque } \\
\text { et al. }\end{array}$ & $\begin{array}{l}\text { Pacientes } \\
\text { com TB }\end{array}$ & $\begin{array}{c}\text { DSS - } \\
\text { econômicos }\end{array}$ & Estudo de coorte & IV & Quantitativo \\
\hline 2007 & Sudeste & Sanches et al. & $\begin{array}{c}\text { População } \\
\text { prisional }\end{array}$ & $\begin{array}{c}\text { DSS - } \\
\text { Violação de } \\
\text { direitos }\end{array}$ & Epidemiológico & IV & Quantitativo \\
\hline 2008 & Sudeste & Diuana et al. & $\begin{array}{c}\text { População } \\
\text { prisional }\end{array}$ & $\begin{array}{c}\text { DSS - } \\
\text { violação de } \\
\text { direitos }\end{array}$ & Pesquisa ação & VI & Qualitativo \\
\hline 2008 & Nordeste & Silva et al. & Idosos & $\begin{array}{c}\text { DSS - } \\
\text { econômicos }\end{array}$ & $\begin{array}{l}\text { Estudo } \\
\text { ecológico }\end{array}$ & IV & Quantitativo \\
\hline
\end{tabular}


R. C. Fiorati et al./ Vittalle v. 30, n. 2 (2018) 59-72

\begin{tabular}{|c|c|c|c|c|c|c|c|}
\hline 2009 & Sudeste & Sanches et al. & $\begin{array}{l}\text { Populações } \\
\text { vulneráveis }\end{array}$ & $\begin{array}{c}\text { Dificuldade } \\
\text { de acesso } \\
\text { aos serviços }\end{array}$ & - & VI & Qualitativo \\
\hline 2009 & $\begin{array}{l}\text { Sudeste/ } \\
\text { Nordeste }\end{array}$ & $\begin{array}{l}\text { Figueiredo } \\
\text { et al. }\end{array}$ & $\begin{array}{c}\text { Doentes } \\
\text { de TB }\end{array}$ & $\begin{array}{c}\text { DSS - } \\
\text { econômicos }\end{array}$ & $\begin{array}{c}\text { Estudo } \\
\text { descritivo } \\
\text { baseado em } \\
\text { inquérito sobre } \\
\text { avaliação de } \\
\text { serviços de } \\
\text { saúde }\end{array}$ & IV & Quantitativo \\
\hline 2009 & Nordeste & Ximenes et al. & $\begin{array}{l}\text { Populações } \\
\text { vulneráveis }\end{array}$ & $\begin{array}{c}\text { DSS - } \\
\text { econômicos }\end{array}$ & $\begin{array}{c}\text { Estudo } \\
\text { caso-controle }\end{array}$ & IV & Quantitativos \\
\hline 2010 & Sudeste & Santos et al. & $\begin{array}{l}\text { Populações } \\
\text { vulneráveis }\end{array}$ & $\begin{array}{c}\text { DSS - } \\
\text { econômicos }\end{array}$ & $\begin{array}{l}\text { Estudo } \\
\text { ecológico }\end{array}$ & IV & Quantitativo \\
\hline 2010 & Nordeste & Nobrega et al. & Indígenas & $\mid \begin{array}{c}\text { Dificuldades } \\
\text { dos serviços de } \\
\text { saúde }\end{array}$ & - & VI & Qualitativo \\
\hline 2010 & Nordeste & $\begin{array}{l}\text { Clementino; } \\
\text { Miranda }\end{array}$ & Doente de TB & $\begin{array}{c}\text { DSS - } \\
\text { econômicos }\end{array}$ & - & VI & Qualitativo \\
\hline 2010 & Sudeste & Queiroz et al. & \begin{tabular}{|c|} 
Pacientes de \\
TB e \\
trabalhadores \\
de Unidades \\
Básicas de \\
Saúde
\end{tabular} & $\begin{array}{c}\text { DSS - } \\
\text { econômicos }\end{array}$ & - & VI & Qualitativo \\
\hline 2010 & Sul & $\begin{array}{c}\text { Bowkalowski; } \\
\text { Bertolozzi }\end{array}$ & $\begin{array}{c}\text { Pacientes } \\
\text { de TB }\end{array}$ & $\begin{array}{c}\text { DSS - } \\
\text { econômicos }\end{array}$ & $\begin{array}{c}\text { Estudo } \\
\text { descritivo e } \\
\text { exploratório }\end{array}$ & IV & Quantitativo \\
\hline 2011 & Sudeste & Arakawa et al. & $\begin{array}{c}\text { Pacientes } \\
\text { de TB }\end{array}$ & $\begin{array}{c}\text { Acessibilidade } \\
\text { aos serviços }\end{array}$ & $\begin{array}{l}\text { Estudo de } \\
\text { avaliação }\end{array}$ & IV & Quantitativo \\
\hline 2011 & Nordeste & $\begin{array}{l}\text { Nogueira } \\
\text { et al. }\end{array}$ & $\begin{array}{c}\text { Profissionais } \\
\text { da saúde }\end{array}$ & $\begin{array}{c}\text { Dificuldades } \\
\text { dos serviços } \\
\text { de saúde }\end{array}$ & $\begin{array}{c}\text { Pesquisa } \\
\text { avaliativa }\end{array}$ & IV & Quantitativo \\
\hline 2011 & Sudeste & $\begin{array}{l}\text { Arcêncio } \\
\text { et al. }\end{array}$ & $\begin{array}{c}\text { Profissionais } \\
\text { da saúde }\end{array}$ & $\begin{array}{c}\text { Dificuldades } \\
\text { dos serviços } \\
\text { de saúde }\end{array}$ & $\begin{array}{c}\text { Estudo } \\
\text { epidemiológico }\end{array}$ & VI & Quantitativo \\
\hline $2011 \mathrm{a}$ & Sudeste & Hino et al. & \begin{tabular}{|c|} 
Pessoas com \\
TB
\end{tabular} & $\begin{array}{c}\text { Dificuldades } \\
\text { dos serviços } \\
\text { de saúde }\end{array}$ & - & VI & Qualitativo \\
\hline $2011 b$ & Sudeste & Hino et al. & $\begin{array}{c}\text { Casos novos } \\
\text { de TB }\end{array}$ & $\begin{array}{c}\text { DSS - } \\
\text { econômicos }\end{array}$ & $\begin{array}{l}\text { Estudo } \\
\text { ecológico }\end{array}$ & IV & Quantitativo \\
\hline 2011 & Sudeste & $\begin{array}{l}\text { Machado } \\
\text { et al. }\end{array}$ & $\begin{array}{c}\text { Pacientes } \\
\text { com TB }\end{array}$ & $\begin{array}{c}\text { DSS - } \\
\text { econômicos }\end{array}$ & $\begin{array}{c}\text { Inquérito } \\
\text { baseado em } \\
\text { questionário }\end{array}$ & IV & Quantitativo \\
\hline 2011 & Sudeste & Oliveira et al. & $\begin{array}{c}\text { Doentes } \\
\text { de TB }\end{array}$ & $\begin{array}{c}\text { Acessibilidade } \\
\text { aos serviços }\end{array}$ & $\begin{array}{c}\text { Estudo } \\
\text { avaliativo }\end{array}$ & IV & Quantitativo \\
\hline 2012 & Nordeste & Alves et al. & $\begin{array}{c}\text { Usuários com } \\
\text { situação de } \\
\text { abandono do } \\
\text { tratamento } \\
\text { da TB }\end{array}$ & $\begin{array}{c}\text { DSS - } \\
\text { econômicos }\end{array}$ & - & VI & Qualitativo \\
\hline 2012 & Sudeste & Beraldo et al. & $\begin{array}{c}\text { Doentes } \\
\text { de TB }\end{array}$ & $\begin{array}{c}\text { Dificuldades } \\
\text { dos serviços } \\
\text { de saúde }\end{array}$ & $\begin{array}{c}\text { Estudo } \\
\text { epidemiológico } \\
\text { transversal }\end{array}$ & IV & Quantitativo \\
\hline
\end{tabular}


Vittalle - Revista de Ciências da Saúde v. 30, n. 2 (2018) 59-72

\begin{tabular}{|c|c|c|c|c|c|c|c|}
\hline 2012 & Nordeste & Souza et al. & $\begin{array}{c}\text { População } \\
\text { prisional }\end{array}$ & $\begin{array}{c}\text { DSS - } \\
\text { Violação } \\
\text { de direitos }\end{array}$ & - & VI & Qualitativo \\
\hline 2012 & Sudeste & Ferreira et al. & $\begin{array}{c}\text { Pacientes } \\
\text { com TB }\end{array}$ & $\begin{array}{c}\text { DSS - } \\
\text { econômicos }\end{array}$ & $\begin{array}{l}\text { Estudo } \\
\text { descritivo } \\
\text { retrospectivo } \\
\text { seccional }\end{array}$ & VI & Quantitativo \\
\hline 2012 & Nordeste & $\begin{array}{l}\text { Souza Filho } \\
\text { et al. }\end{array}$ & $\begin{array}{l}\text { Populações } \\
\text { vulneráveis }\end{array}$ & $\begin{array}{c}\text { DSS - } \\
\text { econômicos }\end{array}$ & - & VI & Qualitativo \\
\hline 2012 & Brasil & $\begin{array}{l}\text { Guimarães } \\
\text { et al. }\end{array}$ & $\begin{array}{l}\text { Populações } \\
\text { vulneráveis }\end{array}$ & $\begin{array}{c}\text { DSS - } \\
\text { econômicos }\end{array}$ & $\begin{array}{l}\text { Estudo } \\
\text { ecológico }\end{array}$ & IV & Quantitativo \\
\hline 2012 & Sudeste & Martinez et al. & Imigrantes & $\begin{array}{c}\text { DSS - } \\
\text { econômicos }\end{array}$ & $\begin{array}{c}\text { Estudo } \\
\text { epidemiológico } \\
\text { descritivo }\end{array}$ & IV & Quantitativo \\
\hline 2012 & Nordeste & $\begin{array}{c}\text { Queiroga } \\
\text { et al. }\end{array}$ & $\begin{array}{c}\text { Casos novos } \\
\text { de TB }\end{array}$ & $\begin{array}{c}\text { DSS - } \\
\text { econômicos }\end{array}$ & $\begin{array}{l}\text { Estudo } \\
\text { ecológico }\end{array}$ & IV & Quantitativo \\
\hline 2012 & Sudeste & $\begin{array}{l}\text { Rodrigues } \\
\text { et al. }\end{array}$ & $\begin{array}{c}\text { Paciente com } \\
\text { TB }\end{array}$ & $\begin{array}{c}\text { DSS - } \\
\text { econômicos }\end{array}$ & $\begin{array}{c}\text { Estudo } \\
\text { transversal }\end{array}$ & IV & Quantitativo \\
\hline 2012 & Sudeste & Roza et al. & $\begin{array}{c}\text { Casos novos } \\
\text { de TB }\end{array}$ & $\begin{array}{c}\text { DSS - } \\
\text { econômicos }\end{array}$ & $\begin{array}{l}\text { Estudo } \\
\text { ecológico }\end{array}$ & IV & Quantitativo \\
\hline 2013 & Brasil & Santos et al. & $\begin{array}{l}\text { Populações } \\
\text { vulneráveis }\end{array}$ & $\begin{array}{c}\text { DSS - } \\
\text { econômicos }\end{array}$ & $\begin{array}{l}\text { Estudo } \\
\text { transversal }\end{array}$ & IV & Quantitativo \\
\hline 2013 & Sudeste & Soares et al. & $\begin{array}{l}\text { Populações } \\
\text { vulneráveis }\end{array}$ & $\begin{array}{c}\text { DSS - } \\
\text { econômicos }\end{array}$ & $\begin{array}{c}\text { Estudo } \\
\text { avaliativo }\end{array}$ & IV & Quantitativo \\
\hline 2013 & Sudeste & Wysocki et al. & $\begin{array}{l}\text { Pacientes } \\
\text { com TB }\end{array}$ & $\begin{array}{c}\text { DSS - } \\
\text { estilo de vida }\end{array}$ & $\begin{array}{c}\text { Estudo } \\
\text { epidemiológico }\end{array}$ & IV & Quantitativo \\
\hline 2013 & Norte & Basta et al. & Indígenas & $\begin{array}{c}\text { DSS - } \\
\text { econômicos }\end{array}$ & $\begin{array}{c}\text { Estudo } \\
\text { epidemiológico }\end{array}$ & IV & Quantitativo \\
\hline 2013 & Norte & $\begin{array}{l}\text { Oliveira; } \\
\text { Gonçalves }\end{array}$ & $\begin{array}{l}\text { Pacientes } \\
\text { com TB }\end{array}$ & $\begin{array}{c}\text { DSS - } \\
\text { econômicos }\end{array}$ & $\begin{array}{c}\text { Estudo } \\
\text { epidemiológico } \\
\text { transversal }\end{array}$ & IV & Quantitativo \\
\hline 2013 & Nordeste & Oliveira et al. & Idosos & $\begin{array}{c}\text { Dificuldades } \\
\text { dos serviços } \\
\text { de saúde }\end{array}$ & - & VI & Qualitativo \\
\hline 2013 & Brasil & Pinheiro et al. & $\begin{array}{l}\text { Paciente } \\
\text { com TB }\end{array}$ & $\begin{array}{c}\text { DSS - } \\
\text { econômicos }\end{array}$ & $\begin{array}{c}\text { Estudo } \\
\text { transversal }\end{array}$ & IV & Quantitativo \\
\hline 2013 & Norte & $\begin{array}{l}\text { Rodrigues } \\
\text { et al. }\end{array}$ & Enfermeiros & $\begin{array}{c}\text { DSS - } \\
\text { econômicos }\end{array}$ & - & VI & Qualitativo \\
\hline 2014 & Sudeste & $\begin{array}{l}\text { Yamamura } \\
\text { et al. }\end{array}$ & $\begin{array}{l}\text { Populações } \\
\text { vulneráveis }\end{array}$ & $\begin{array}{c}\text { Dificuldades } \\
\text { dos serviços } \\
\text { de saúde }\end{array}$ & $\begin{array}{l}\text { Estudo } \\
\text { ecológico }\end{array}$ & IV & Quantitativo \\
\hline 2014 & Brasil & $\begin{array}{l}\text { Harling; } \\
\text { Castro }\end{array}$ & $\begin{array}{c}\text { Casos de } \\
\text { notificação } \\
\text { de TB }\end{array}$ & $\begin{array}{c}\text { DSS - } \\
\text { econômicos }\end{array}$ & $\begin{array}{l}\text { Estudo } \\
\text { ecológico }\end{array}$ & IV & Quantitativo \\
\hline
\end{tabular}

O número de artigos publicados por anos está representado na figura $2 \mathrm{~A}$, com destaque para o ano de 2012, que apresenta nove artigos publicados $(20,0 \%)$.

Em relação à região de estudo, a figura 2B apresenta a frequência dos estudos cuja abrangência era nacional e dividida por macrorregiões. 

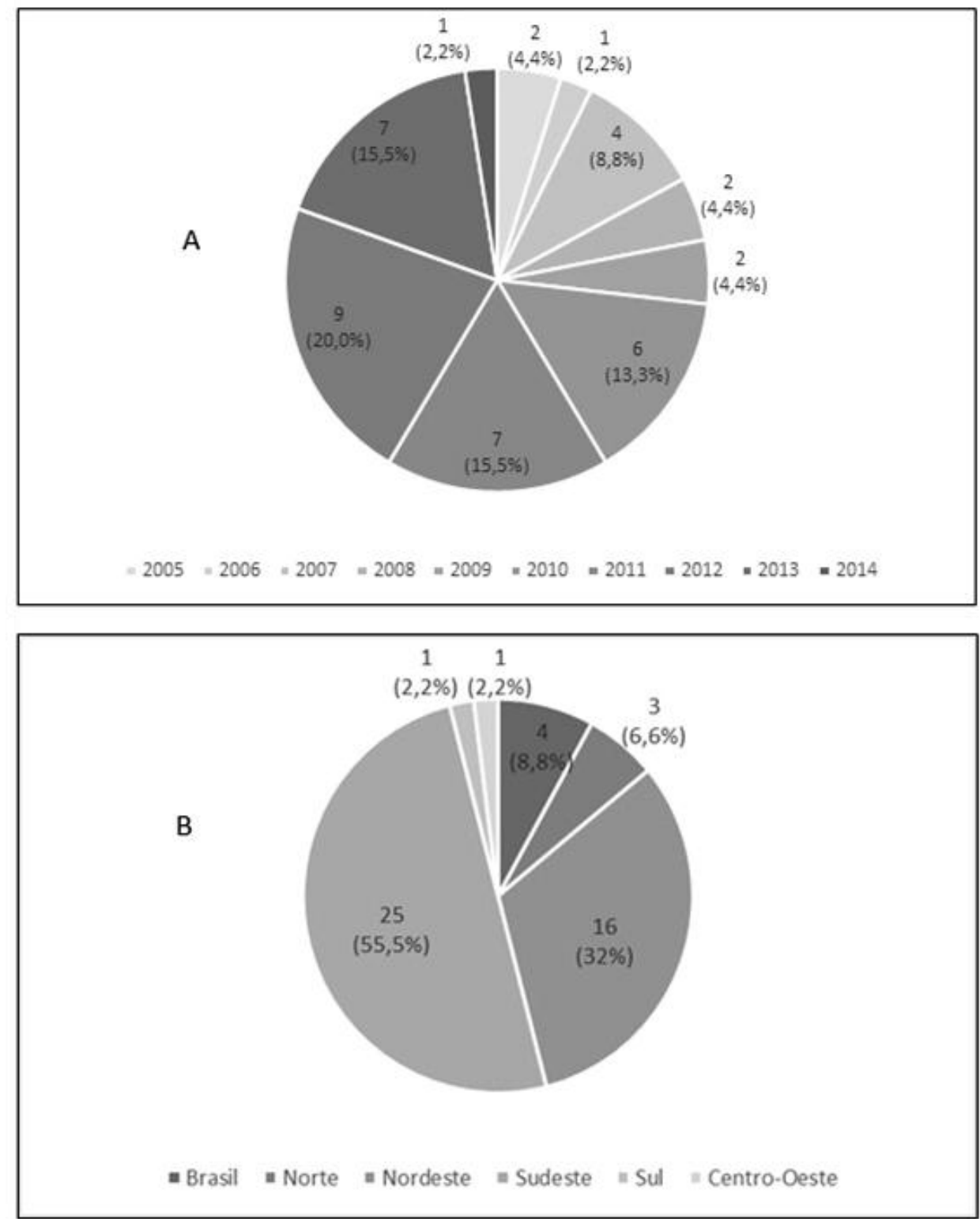

Figura 2: Distribuição dos estudos segundo ano e macrorregiões brasileiras.

No que diz respeito ao delineamento metodológico, 33 (73,3\%) eram estudos observacionais e $12(26,6 \%)$ com abordagem qualitativa.

Quanto à classificação da hierarquia do nível evidência dos manuscritos, 31 (68,9\%) foram classificados no nível de evidência IV e $14(31,1 \%)$ classificados no nível VI.

Com relação às populações e/ou amostras, três $(6,6 \%)$ estudos constituíram-se de população em regime de privação de liberdade; um (2,2\%) com populações de imigrantes e três $(6,6 \%)$ com populações indígenas. Houve ainda $27(60 \%)$ estudos desenvolvidos com populações adscritas a serviços de saúde da Estratégia de Saúde da Família (ESF). Os estudos desenvolvidos junto às populações vulneráveis habitantes de territórios urbanos periféricos e alto índice de desigualdades sociais constituíram-se em oito $(17,7 \%)$. Por fim, os estudos desenvolvidos com populações de idosos somaram três $(6,6 \%)$.

No que tange à temática abordada, quatro $(8,8 \%)$ atribuem a acessibilidade aos serviços de saúde como principal problema relacionado às desigualdades em saúde; oito $(17,7 \%)$ dos estudos atribuem às deficiências apresentadas pelos serviços de saúde, envolvendo as desigualdades em saúde vividas pela população pesquisada como: organização dos serviços, estratégia deficitária dos profissionais e incapacidade para 
verificar as necessidades sociais dos usuários e estigma social apresentado pelos profissionais, assinala-se, ainda, que todos esses fatores foram apontados como determinantes para o atraso do diagnóstico e do tratamento da TB em tempo oportuno.

Da amostra, $33(73,3 \%)$ dos artigos afirmam que as desigualdades em saúde como fator interveniente na cadeia de progressão da TB se devem a fatores relacionados aos Determinantes Sociais da Saúde (DSS), interferindo no acesso aos serviços de saúde e também no próprio desfecho não favorável, como abandono e morte por TB. Observouse, ainda, que 38 estudos $(84,8 \%)$ atribuem às causas econômicas as desigualdades vividas pela população diagnosticada com TB; quatro $(9,0 \%)$ referem-se à violação de direitos, estudos que se relacionaram com população em regime de privação de liberdade; e, ainda, três $(6,0 \%)$ dos artigos atribuem as desigualdades a fatores ligados ao estilo de vida dos doentes diagnosticados com a doença, sendo que, em todos os estudos computados, o estilo de vida apontado é aquele relacionado ao uso de álcool e outras drogas.

Os resultados mostram que algumas temáticas (Quadro 5) são apontadas como fatores determinantes das condições de desigualdade social que impactam a cadeia de progressão da TB nas populações dos estudos levantados. Em relação ao processo de progressão da doença: infecção, adoecimento, acesso ao tratamento e cura, os artigos referem que essa progressão está relacionada a insalubridade dos domicílios, a pobreza extrema, a falta de oportunidades ou de trabalho, as condições de trabalho e o não capital social das comunidades afetadas pela TB. Observou-se, ainda, que algumas políticas públicas vêm sendo implementadas, como o Programa Bolsa Família, no entanto, há carência de estudos que possam evidenciar o impacto dessas políticas na cadeia de progressão da TB.

Quadro 5: Determinantes que impactam na cadeia de progressão da tuberculose

\begin{tabular}{|c|c|c|}
\hline Temáticas & Fatores determinantes & Estudos \\
\hline $\begin{array}{c}\text { Determinantes } \\
\text { relacionados à } \\
\text { organização dos } \\
\text { serviços de saúde }\end{array}$ & $\begin{array}{c}\text { Distância geográfica, horário de funcionamento dos serviços, } \\
\text { falta de insumos (pote de escarro, geladeira para } \\
\text { acondicionamento do material biológico, sistema de registro } \\
\text { e/ou informação, retaguarda laboratorial, sistema de apoio), não } \\
\text { política institucional de acolhimento do Sintomático } \\
\text { Respiratório em situação de vulnerabilidade e o estigma social } \\
\text { manifestado por profissionais }\end{array}$ & 12 \\
\hline \multirow{2}{*}{$\begin{array}{c}\text { Determinantes sociais } \\
\text { de saúde }\end{array}$} & Econômicos & 28 \\
\cline { 2 - 3 } & Violação de direitos & 03 \\
\hline Total & Culturais e estilo de vida & 02 \\
\hline
\end{tabular}

\section{Discussão}

Baseado no objetivo do estudo, verificou-se que poucos estudos, principalmente aqueles publicados anteriormente ao ano de 2008, têm utilizado como descritor determinantes sociais, o que demonstra os limites da produção de conhecimento nessa área no país.

Embora tais estudos não tenham utilizado esse descritor, abordaram os determinantes como recorte para cotejo dos resultados. Os estudos trouxeram os seguintes fatores associados à cadeia de progressão da TB: determinantes socioeconômicos; a organização dos serviços de saúde como obstáculo à acessibilidade ao diagnóstico e ao tratamento; violação de direitos, fatores culturais e de estilo de vida.

Em relação ao referencial teórico utilizado (10), verificou-se que os artigos focaram as desigualdades sociais com base nas desigualdades econômicas como principal fator de 
impacto na cadeia de progressão da TB no Brasil. Assim, evidencia-se que os estudos têm-se centrado nos determinantes estruturais, que são aqueles ligados à estratificação social e incluem os fatores socioeconômicos, trabalho e renda, gênero, etnicidade, cultura e redes de apoio social $(8,10)$. Esses estudos investiram em populações especificamente vulneráveis, como idosos, pessoas habitantes de periferias urbanas e regiões geográficas com índices altos de pobreza, pessoas em regime de privação de liberdade, imigrantes de países sul-americanos e indígenas.

Entretanto, também se observaram estudos que mostraram que determinantes, como acesso a serviços de saúde, qualidade e organização dos serviços referente à Atenção Primária à Saúde (APS) no âmbito da ESF, não responderam em tempo oportuno e de forma adequada às reais necessidades sociais e de saúde de sua população adscrita diagnosticadas com TB. Preconceitos manifestados por profissionais de saúde, estilos de vida e comportamentos também se caracterizam como determinantes sociais importantes ao aumento de TB em determinadas regiões do país, podendo classificá-los como determinantes intermediários (11) segundo o referencial teórico (10).

Em relação às temáticas abordadas, a maioria dos estudos que atribui aos DSS e a desigualdade social vivida por pessoas diagnosticadas pela doença foram publicados entre os anos de 2009 e 2014, sendo que, no período de 2005 a 2008, os artigos dão ênfase ao acesso e a deficiências dos serviços em responder às necessidades dos pacientes, o que tende a levar a interrupções e ao abandono. Esse fato pode estar associado a um maior investimento histórico em estudos e estratégias de enfrentamento aos DSS e às desigualdades sociais em saúde desenvolvidas pela OMS e os estímulos endereçados a governos nacionais para formularem políticas sociais e de saúde, visando à superação das injustiças sociais e seus impactos na saúde (17).

Um fator importante associado ao impacto de iniquidades na cadeia de progressão da TB foi a violação de direitos contra a população em situação de privação de liberdade residente em prisões do país, os quais têm a acessibilidade ao diagnóstico e ao tratamento da doença condicionados pelos agentes carcerários, com a conivência da administração dos presídios, os quais utilizam como moeda de negociação com os presos o direito à consulta médica diante da suspeita de TB. Assim, os presos que "cooperam" com o sistema carcerário recebem o direito à consulta e à realização de exames necessários ao diagnóstico, mas aos que não "cooperam", o acesso à consulta é retardado, intencionalmente, pelos agentes carcerários. Embora, certamente, todas as desigualdades referidas nesse estudo constituem igualmente violações de direitos, nesses casos, essa violação incorre em crime hediondo, mas silenciado pela omissão do Estado e das instâncias jurídicas do país.

Além disso, os estudos concordam na análise do fenômeno e afirmam que esse comportamento dos agentes prisionais está associado a determinadas representações sociais e ideológicas presentes e referendadas por todo o imaginário social brasileiro, de que essa população não compartilha da mesma condição humana que o resto da sociedade. São vistos e representados, portanto, como destituídos de humanidade, ou em uma posição de subumanidade em comparação com o restante das pessoas $(18,19)$.

A análise mais profunda desse fenômeno associa-se a uma cultura nacional, a qual hierarquiza em estratos sociais toda a sociedade, disponibilizando, de forma desigual, os direitos humanos e sociais, assim como a cidadania é exercida de uma forma regulada e não plena, sendo que, para alguns, os direitos são exercidos de forma plena, enquanto, para outros, a exclusão dos direitos humanos, sociais e políticos são marcadamente um acontecimento cotidiano (20,21).

Agrega-se, ainda, outro fator significativo para essa análise, que é a presença na cultura brasileira de manifestações da ordem social e econômica escravagista, 
prevalente no Brasil até o século XIX, mas que até a contemporaneidade se revela segmentadora de grupos sociais para uma situação de apartação social, dentro de uma cultura de naturalização da pobreza, o que determina um comportamento social de negligência em relação a segmentos sociais excluídos (22).

Outro dado interessante a assinalar são os estudos que apontaram os estilos de vida ligados ao uso de álcool e de outras drogas como diretamente responsáveis por índices de abandono, MDR e morte por TB. Estudos atrelando fatores culturais e estilos de vida têm sido comuns na literatura referente às desigualdades sociais em saúde. Algumas críticas são feitas a essa abordagem, as quais têm apontado que atribuir às desigualdades sociais em saúde estilos de vida de determinados grupos sociais e/ou culturais tende a minimizar outros fatores que podem estar fundamentalmente na base das desigualdades como os fatores socioeconômicos, políticos, que permeiam determinadas sociedades (23-25).

Assim, ao se desconsiderar as desigualdades econômicas de exclusão social a que determinados grupos sociais estão expostos, obscurece-se a análise que aponta como a forma em que uma sociedade se organiza é que tem maior impacto sobre processos de saúde-doença em comparação com comportamentos individuais. Como exemplo, vê-se que as condições iníquas de vida da população que reside em áreas periféricas urbanas próximas a pontos de tráfico de drogas, bem como sua associação frequente com facções do crime organizado ligado ao tráfico de drogas, tornam-nas vulneráveis ao uso de álcool e de outras drogas muito mais do que outros grupos sociais, determinando, assim, estilos pouco saudáveis de vida. Além disso, a condição de vida dessa população, mais vulnerável ao uso de substâncias, resulta, frequentemente, em expulsão para a situação de rua e, consequentemente, na perda de vínculos sociais e acessos inclusive a serviços de saúde e aos tratamentos $(26,27)$.

Os contextos sociais e políticos determinam fortemente processos de saúde/doença em certos segmentos populacionais e esses são os desafios à estratégia de eliminação da doença no Brasil. As formas de enfrentamento das desigualdades constituem os principais focos de atenção em cada país ou região. Assim, as diretrizes de ação devem focar-se em áreas de impacto direto como exclusão social e gênero; inclusão de perspectivas para toda a vida; desenvolvimento na infância; foco nas áreas ligadas aos Sistemas de Saúde; foco na vulnerabilidade e na alta exposição como condições de trabalho e moradia; foco no rápido crescimento urbano, como assentamentos urbanos e regiões perifericamente excluídas da mobilidade urbana e de serviços necessários à boa qualidade de vida.

Outro fator importante que merece destaque é a globalização da economia como macrodeterminante da condição mundial que tem afetado conjunturas nacionais, levando a crises econômicas insuportáveis e à vigência de governos nacionais comprometidos com o capital mundial e com as fortes economias do Norte, descomprometendo-se com as políticas sociais $(22,24)$.

Para sumarizar, verificou-se que as pesquisas analisadas mostraram que os principais fatores que associam as desigualdades à TB se referem aos fatores econômicos, organização dos serviços de saúde e a sua forma de funcionamento, assim como a fatores geográficos que dificultam a acessibilidade aos serviços, seguidos de situações marcadas por violações de direitos referentes a populações vulneráveis e a estilos de vida.

Dessa forma, considerando a pergunta orientadora da revisão de literatura qualitativa, o objetivo geral do estudo e a análise dos resultados contemplados pelos estudos analisados verificam tais fatores como principais associados ao impacto das desigualdades sociais no contexto da TB no Brasil e os desafios à sociedade brasileira ainda são substanciais e devem envolver, para além do setor da saúde, todos os setores 
ligados à gestão pública nas várias esferas governamentais do país. A intersetorialidade e as políticas públicas intervenientes nos determinantes estruturais e intermediários devem ser construídas em diversos níveis, desde o superestrutural, envolvendo a fusão e/ou cooperação de Ministérios em prol da erradicação da pobreza e desigualdades sociais como prioridades nacionais (17).

Reforça-se a importância da proteção social como uma garantia de inclusão de todos os cidadãos em situação de vulnerabilidade social e/ou situação de risco, tendo como objetivo prevenir situações de risco por meio de redes de apoio social e fortalecimento de vínculos familiares e/ou comunitários (28).

Algumas limitações desse trabalho devem ser apontadas como a heterogeneidade e abrangência dos estudos; o próprio conceito de desigualdades sociais é abstrato e de difícil tradução em descritores controlados; a exclusão de outros tipos de estudos que não puderam ser classificados nos níveis de evidência determinados pelo referencial, tais como teses, documentos governamentais, revisões de literatura, entre outros.

Estudos futuros poderiam explorar, com maior profundidade, aspectos que ficaram limitados neste estudo, tais como as ações e estratégias que foram e vêm sendo desenvolvidas com o intuito de enfrentamento às desigualdades sociais que impactam a cadeia de progressão da TB no Brasil.

Como todo estudo de revisão de literatura, há o viés da publicação, que ocorre pela não inclusão de todas as investigações desenvolvidas sobre o tema, e somente aqueles publicados, que tendem a apresentar resultados positivos do ponto de vista estatístico. A não consideração dos estudos não publicados e ou com resultado negativo podem trazer esse viés à pesquisa. Além disso, em face da heterogeneidade dos estudos, conduziu-se uma revisão de literatura qualitativa, em que o nível de evidência é menor que a metanálise.

\section{Conclusão}

Em conclusão, por meio da revisão de literatura, pode-se observar que a eliminação da TB não é um processo simples, o arsenal necessário à sua concretude transcende a dimensão tecnológica médica ou biomédica e deve estar alicerçado e potencializado por uma compreensão mais profunda sobre as questões sócio-históricas do Brasil. A exclusão, a desigualdade e a pobreza são obstáculos importantes à eliminação da doença, portanto as intervenções intersetoriais para além da saúde devem ser de amplo espectro e alavancar o Estado do Bem-Estar Social que reza na Constituição, uma reforma social de larga envergadura, necessária para o cumprimento do ideário de um mundo sem TB.

CONFLITO DE INTERESSES: os autores declaram não haver conflito de interesses.

\section{Referências}

1. World Health Organization. Global Tuberculosis Report 2016. Geneva: WHO; 2016.

2. WHO. End TB Strategy 2015. Geneva: World Health Organization; 2015. Avaiable: http://www.who.int/tb/post2015_strategy/en/. Accessed 9 January 2016.

3. Maciel EL, Reis-Santos B. Determinants of tuberculosis in Brazil: from conceptual framework to practical application. Rev Panam Salud Publica 2015, 38(1):28-34

4. Huynh G. Can China achieve the WHO global targets for TB control by 2035? Trans R Soc Trop Med Hyg 2016; 110: 161-62. 
5. Lönnroth K, Raviglione M. The WHO's new End TB Strategy in the post-2015 era: working with the Sustainable Development Goals Trans R Soc Trop Med Hyg 2016; 110:148-50.

6. Gesesew H, Tsehaineh B, Massa D, Tesfay A, Kahsay Hafte, Mwanri L. The role of social determinants on tuberculosis/HIV co-infection mortality in southwest Ethiopia: a retrospective cohort study. BMC Res Notes 2016; 9(1): 89.

7. Odone A, Calderon R, Becerra MC, Zhang Z, Contreras CC, Yataco R et al. Acquired and Transmitted Multidrug Resistant Tuberculosis: The Role of Social Determinants. PLoS One 2016; 11(1): e0146642.

8. Carvalho AI. Determinantes sociais, econômicos e ambientais da saúde. In Fundação Oswaldo Cruz. A saúde no Brasil em 2030 - prospecção estratégica do sistema de saúde Brasileiro : população e perfil sanitário [online]. Rio de Janeiro: Fiocruz/Ipea/Ministério da Saúde/Secretaria de Assuntos Estratégicos da Presidência da República, 2013; 2: 19-38.

9. Hargreaves JR, Boccia D, Evans CA, Adato M, Petticrew M, Porter JDH. The social determinants of tuberculosis: from evidence to action. Am J Public Health 2011; 101: 654-62.

10. WHO. Comission on Social Determinants of Health 2008. Closing the gap in a generation: health equity through action on the social determinants of health. Final Report of the Commission on Social Determinants of Health. Geneva: World Health Organization, 2008.

11. Buss PM, Pellegrini-Filho A. A saúde e seus determinantes sociais. PHYSIS: Rev. Saúde Coletiva, Rio de Janeiro, 2007; 17(1):77-93.

12. Sampaio RF, Mancini MC. Estudos de revisão sistemática: um guia para síntese criteriosa da evidência científica. Rev. bras. fisioter. 2007, 11(1): 83-9.

13. Malik AM. How to write na introduction and methods of a systematic review of literature. J Pak Med Assoc. 2014,64(10). Avaiable: http://ecommons.aku.edu/pakistan_fhs_mc_chs_chs

14. Cooke A, Smith D, Booth A. Beyond PICO The SPIDER tool for qualitative evidence synthesis. Qual Health Res. 2012; 22: 1435-43.

15. Galvão CM, Sawada NO, Trevisan MA. Revisão sistemática: recurso que proporciona a incorporação das evidências na prática da enfermagem. Rev Latino-am Enfermagem 2004, 12(3): 549-56.

16. Melnyk BM. Advancing Evidence-Based Practice in Clinical and Academic Settings. Worldviews on Evidence-Based Nursing 2005, 2(3): 161-65.

17. Segura del Pozo JS. Desigualdades sociales en salud: conceptos, estúdios e intervenciones (19802010). Bogotá. [Doctora] - Universidad Nacional de Colombia, Interfaculdades em Salud Pública, 2013.

18. Sánchez AR, Massari V, Gerhardt G, Barreto AW, Cesconi V, Pires J et al . A tuberculose nas prisões do Rio de Janeiro, Brasil: uma urgência de saúde pública. Cad. Saúde Pública 2007; 23(3): 545-52.

19. Diuana V, Lhuilier D, Sánchez AR, Amado G, Araújo L, Duarte AM et al . Saúde em prisões: representações e práticas dos agentes de segurança penitenciária no Rio de Janeiro, Brasil. Cad. Saúde Pública 2008; 24(8): 1887-96.

20. Souza KMJ, Villa TCS, Assolini FEP, Beraldo AA, França UM, Protti ST et al. Atraso no diagnóstico da tuberculose em sistema prisional: a experiência do doente apenado. Texto contexto enferm. 2012; 21(1): 17-25.

21. Holston J. Cidadania insurgente: disjunções da democracia e da modernidade no Brasil. São Paulo: Companhia das Letras, 2013.

22. Accorssi A, Scarparo H, Guareschi P. A naturalização da pobreza: reflexões sobre a formação do pensamento social. Psicologia \& Sociedade, 2012; 24(3): 536-46.

23. Almeida-Filho N. Modelos de determinação social das doenças crônicas não-transmissíveis. Ciênc. saúde coletiva 2004; 9(4): 865-84.

24. Marmot M. Social determinants of health inequalities. Lancet 2005; 365(9464): 1099-104.

25. Krieger N, Dorling D, McCartney G. Mapping injustice, visualizing equity: Why theory, metaphors and images matter in tackling inequalities. Public Health 2012; 126(3): 256- 8

26. Alves VS. Modelos de atenção à saúde de usuários de álcool e outras drogas: discursos políticos, saberes e práticas. Cad. Saúde Pública 2009; 25(11): 2309-19. 
27. Adorno RCF. Atenção à saúde, direitos e o diagnóstico como ameaça: políticas públicas e as populações em situação de rua. Etnografica 2011; 15(3): 543-67.

28. Centre on Global Health Security. Social Protection Interventions for Tuberculosis Control: The Impact, the Challenges, and the Way Forward. Available:https://www.chathamhouse.org/sites/files/chathamhouse/public/Research/Global\%20Healt h/170212summary.pdf 